\title{
ON GENERALIZATION OF PALEY-WIENER THEOREM FOR WEIGHTED HARDY SPACES
}

\author{
B.V. VINNITSKII, V.N. DILNYI
}

\begin{abstract}
We consider the Hardy space $H_{\sigma}^{p}\left(\mathbb{C}_{+}\right)$in the half-plane with an exponential weight. In this space we study the analytic continuation from the boundary. In the previous works for the case $p \in(1,2]$ a result on analytic continuation from the imaginary axis was obtained, and it was a generalization of Paley-Wiener theorem. But for many applications the case $p=1$ is more interesting. For this case in the paper we obtain estimates for a function satisfying certain standard conditions.
\end{abstract}

Keywords: weighted Hardy space, Paley-Wiener theorem, angular boundary values.

Mathematics Subject Classification: $30 \mathrm{H} 10,30 \mathrm{E} 20$

\section{INTRODUCTION}

We denote by $H^{p}\left(\mathbb{C}_{+}\right), 1 \leqslant p<+\infty$, the space of functions analytic in $\mathbb{C}_{+}=\{z: \operatorname{Re} z>0\}$ obeying

$$
\|f\|_{H^{p}}:=\sup _{-\frac{\pi}{2}<\varphi<\frac{\pi}{2}}\left\{\int_{0}^{+\infty}\left|f\left(r e^{i \varphi}\right)\right|^{p} d r\right\}^{1 / p}<+\infty .
$$

A.M. Sedletskii [1] showed that this space coincides with Hardy space $\widetilde{H}^{p}\left(\mathbb{C}_{+}\right)$of analytic in $\mathbb{C}_{+}$functions for which

$$
\|f\|_{\widetilde{H}^{p}}=\sup _{x>0}\left\{\int_{-\infty}^{+\infty}|f(x+i y)|^{p} d y\right\}^{1 / p}<+\infty,
$$

and the norms $\|\cdot\|_{H^{p}}$ and $\|\cdot\|_{\widetilde{H}^{p}}$ are equivalent. The properties of Hardy space are described rather in detail in [2], [3]. The functions in Hardy space have angular boundary values almost everywhere on $i \mathbb{R}$ which we indicate by $f(i y)$ and $f(i y) \in L^{p}(\mathbb{R})$. The following Paley-Wiener theorem is well-known [3, p. 94].

Theorem 1. Function $f_{0} \in L^{p}(i \mathbb{R}), 1 \leqslant p \leqslant 2$, is an angular boundary function of some function $f \in H^{p}\left(\mathbb{C}_{+}\right)$if and only if

$$
\int_{-\infty}^{+\infty} f_{0}(i t) e^{i \tau t} d t=0
$$

for almost all negative numbers $\tau$.

B.V. Vinnitskit, V.N. Dilnyi, On generalization of Paley-Wiener theorem for weighted HARDY SPACES.

(C) Vinnitski B.V., Dilnyi V.N. 2013.

Submitted September 28, 2013. 
B.V. Vinnitskii considered [4] the following generalization of Hardy space. We denote by $H_{\sigma}^{p}\left(\mathbb{C}_{+}\right), \sigma \geqslant 0,1 \leqslant p<+\infty$, the space of analytic in $\mathbb{C}_{+}$function for which

$$
\|f\|_{H_{\sigma}^{p}}:=\sup _{-\frac{\pi}{2}<\varphi<\frac{\pi}{2}}\left\{\int_{0}^{+\infty}\left|f\left(r e^{i \varphi}\right)\right|^{p} e^{-p r \sigma|\sin \varphi|} d r\right\}^{1 / p}<+\infty .
$$

The functions in this space have angular boundary values almost everywhere on $i \mathbb{R}$ which we denote by $f(i y)$ and $f(i y) e^{-\sigma|y|} \in L^{p}(\mathbb{R})$.

The following generalization of Paley-Wiener theorem was obtained in [5].

Theorem 2. A function $f_{0}: i \mathbb{R} \rightarrow \mathbb{C}$ such that $f_{0}(i t) e^{-\sigma|t|} \in L^{p}(\mathbb{R}), 1<p \leqslant 2$, is an angular boundary function of a function $f \in H_{\sigma}^{p}\left(\mathbb{C}_{+}\right)$if and only if there exists a function $f_{2}$ such that

1) $f_{2} \in H_{2 \sigma}^{p}\left(\mathbb{C}_{+}\right)$;

2) $f_{3}(i v):=f_{1}(i v)+f_{2}(i v) \in L^{p}(-\infty ; 0), f_{1}(i v):=f_{0}(i v) e^{-\sigma v}$;

3) for almost each $\tau<0$

$$
\int_{0}^{+\infty} f_{1}(i v) e^{i \tau v} d v+\frac{1}{i} \int_{0}^{+\infty} f_{2}(u) e^{\tau u} d u+\int_{-\infty}^{0} f_{3}(i v) e^{i \tau v} d v=0 .
$$

Theorem 1 is a particular case of Theorem 2 for the case $\sigma=0$. In studies of cyclicity in some spaces and the properties of convolution equations (see [6], [7]) there appear an issue of validity of Theorem 2 for the case $p=1$. It was shown in [5] that the necessary conditions of this theorem are true also for the case $p=1$, but the question on sufficiency remains open. The aim of this paper is to describe the functions whose angular boundary functions satisfy conditions 1)-3) of the previous theorem.

\section{MAIN RESULT}

We obtain the following statement.

Theorem 3. If $f_{0}: i \mathbb{R} \rightarrow \mathbb{C}, f_{0}(i t) e^{-\sigma|t|} \in L^{1}(\mathbb{R})$ and conditions 1)-3) of Theorem 2 are satisfied, then there exists analytic in $\mathbb{C}_{+}$function $f$, for which function $f_{0}$ is an angular boundary function and

$$
\sup \left\{\int_{0}^{+\infty}\left|f\left(r e^{i \varphi}\right)\right| e^{-\sigma r|\sin \varphi|} d r: \varphi \in(-\pi / 2 ; \pi / 2) \backslash(-\delta ; \delta)\right\}<+\infty
$$

for each $\delta \in(0 ; \pi / 2)$.

The proof is based mainly on the following auxiliary statements.

Lemma 1. If the hypothesis of Theorem 3 holds and

$$
\Xi(z):=\frac{1}{2 \pi} \int_{0}^{+\infty} \frac{f_{1}(i v)}{i v-z} d v+\frac{1}{2 \pi} \int_{-\infty}^{0} \frac{f_{3}(i v)}{i v-z} d v+\frac{1}{2 \pi i} \int_{0}^{+\infty} \frac{f_{2}(u)}{u-z} d u,
$$

then $\Xi(z)=0$ for each $z \in \mathbb{C}_{-}:=\{z: \operatorname{Re} z<0\}$.

Proof. We denote

$$
\eta_{1}(\tau)=\int_{0}^{+\infty} f_{1}(i v) e^{i \tau v} d v, \eta_{2}(\tau)=\frac{1}{i} \int_{0}^{+\infty} f_{2}(u) e^{\tau u} d u, \eta_{3}(\tau)=\int_{-\infty}^{0} f_{3}(i v) e^{i \tau v} d v .
$$


Then it follows from Condition 3) that

$$
\int_{-\infty}^{0} e^{-\tau z}\left(\eta_{1}(\tau)+\eta_{2}(\tau)+\eta_{3}(\tau)\right) d \tau=0, \quad \operatorname{Re} z<0
$$

But by Fubini theorem we have for $\operatorname{Re} z<0$

$$
\int_{-\infty}^{0} e^{-\tau z} \eta_{1}(\tau) d \tau=\int_{0}^{+\infty} f_{1}(i v) \int_{-\infty}^{0} e^{\tau(i v-z)} d \tau d v=\int_{0}^{+\infty} \frac{f_{1}(i v)}{i v-z} d v .
$$

In the same way

$$
\int_{-\infty}^{0} e^{-\tau z} \eta_{3}(\tau) d \tau=\int_{-\infty}^{0} \frac{f_{3}(i v)}{i v-z} d v, \quad \int_{-\infty}^{0} e^{-\tau z} \eta_{2}(\tau) d \tau=\frac{1}{i} \int_{0}^{+\infty} \frac{f_{2}(u)}{u-z} d u .
$$

This is why by (2) we complete the proof.

We introduce the notation $\mathbb{C}(\alpha ; \beta)=\{z: \alpha<\arg z<\beta\}$.

Lemma 2. If the hypothesis of Theorem 3 holds, then the angular boundary values of the function

$$
f(z)=\left\{\begin{array}{l}
-e^{-i \sigma z} \Xi(z), z \in \mathbb{C}(0 ; \pi / 2), \\
-e^{-i \sigma z}\left(\Xi(z)-f_{2}(z)\right), z \in \mathbb{C}(-\pi / 2 ; 0),
\end{array}\right.
$$

coincide with function $f_{0}$ almost everywhere on $i \mathbb{R}$.

Proof. By Lemma 1 we have $\Xi(-\bar{z})=0, z \in \mathbb{C}_{+}$. This is why

$$
\begin{aligned}
\Xi(z)= & \Xi(z)-\Xi(-\bar{z})=\frac{1}{2 \pi} \int_{0}^{+\infty} f_{1}(i v)\left(\frac{1}{i v-z}-\frac{1}{i v+\bar{z}}\right) d v \\
& +\frac{1}{2 \pi} \int_{-\infty}^{0} f_{3}(i v)\left(\frac{1}{i v-z}-\frac{1}{i v+\bar{z}}\right) d v+\frac{1}{2 \pi i} \int_{0}^{+\infty} f_{2}(u)\left(\frac{1}{u-z}-\frac{1}{u+\bar{z}}\right) d u \\
= & \frac{1}{2 \pi} \int_{0}^{+\infty} f_{1}(i v) \frac{2 x}{(i v-z)(i v+\bar{z})} d v+\frac{1}{2 \pi} \int_{-\infty}^{0} f_{3}(i v) \frac{2 x}{(i v-z)(i v+\bar{z})} d v \\
& +\frac{1}{2 \pi i} \int_{0}^{+\infty} f_{2}(u) \frac{2 x}{(u-z)(u+\bar{z})} d u .
\end{aligned}
$$

The latter integral vanishes [1] on the imaginary axis except possibly the point $z=0$. It is easy to see that

$$
\frac{1}{2 \pi} \int_{0}^{+\infty} f_{1}(i v) \frac{2 x}{(i v-z)(i v+\bar{z})} d v=-\frac{1}{\pi} \int_{0}^{+\infty} f_{1}(i v) \frac{x}{(v-y)^{2}+x^{2}} d v
$$

is the Poisson integral and thus it possesses angular boundary values in $\mathbb{C}_{+}$almost everywhere on $\partial \mathbb{C}_{+}$and these values are equal to $-f_{1}(i v)$ for $v>0$ and 0 for $v<0$. In the same way one can show that angular boundary values in $\mathbb{C}_{+}$on $\partial \mathbb{C}_{+}$of the function

$$
\frac{1}{2 \pi} \int_{-\infty}^{0} f_{3}(i v) \frac{2 x}{(i v-z)(i v+\bar{z})} d v
$$


are equal to $-f_{3}(i v)$ for $v<0$ and vanish for $v>0$ almost everywhere.

Lemma 3. If the hypothesis of Theorem 3 holds, then the function $f$ defined in Lemma 2 satisfies condition (1).

Proof. It follows from Lemma 1 that for $\operatorname{Re} z>0$

$$
\begin{aligned}
& 0=\frac{1}{2 \pi} \int_{0}^{+\infty} \frac{f_{1}(i v)}{i v+z} d v+\frac{1}{2 \pi} \int_{-\infty}^{0} \frac{f_{3}(i v)}{i v+z} d v+\frac{1}{2 \pi i} \int_{0}^{+\infty} \frac{f_{2}(u)}{u+z} d u, \\
& 0=\frac{1}{2 \pi} \int_{0}^{+\infty} \frac{f_{1}(i v)}{i v+\bar{z}} d v+\frac{1}{2 \pi} \int_{-\infty}^{0} \frac{f_{3}(i v)}{i v+\bar{z}} d v+\frac{1}{2 \pi i} \int_{0}^{+\infty} \frac{f_{2}(u)}{u+\bar{z}} d u .
\end{aligned}
$$

By the above identities we obtain that for $z=x+i y \in \mathbb{C}_{+}, y \neq 0$,

$$
\begin{aligned}
0= & \frac{1}{2 \pi} \int_{0}^{+\infty} f_{1}(i v)\left(-\frac{1}{i v+\bar{z}}+\frac{x}{i y}\left(\frac{1}{i v+\bar{z}}-\frac{1}{i v+z}\right)\right) d v \\
& +\frac{1}{2 \pi} \int_{-\infty}^{0} f_{3}(i v)\left(-\frac{1}{i v+\bar{z}}+\frac{x}{i y}\left(\frac{1}{i v+\bar{z}}-\frac{1}{i v+z}\right)\right) d v \\
& +\frac{1}{2 \pi i} \int_{0}^{+\infty} f_{2}(u)\left(-\frac{1}{u+\bar{z}}+\frac{x}{i y}\left(\frac{1}{u+\bar{z}}-\frac{1}{u+z}\right)\right) d u .
\end{aligned}
$$

Hence, denoting

$$
\chi(w ; z):=\frac{2}{\pi i} \frac{w x}{(w+\bar{z})(w-z)(w+z)}=\frac{1}{2 \pi i}\left(\frac{1}{w-z}-\frac{1}{w+\bar{z}}+\frac{x}{i y}\left(\frac{1}{w+\bar{z}}-\frac{1}{w+z}\right)\right),
$$

we obtain

$$
\Xi(z)=i \int_{0}^{+\infty} f_{1}(i v) \chi(i v ; z) d v+i \int_{-\infty}^{0} f_{3}(i v) \chi(i v ; z) d v+\int_{0}^{+\infty} f_{2}(u) \chi(u ; z) d u .
$$

First we consider the case when $0<\varphi<\pi / 2$. Then

$$
\begin{aligned}
\int_{0}^{+\infty}\left|f\left(r e^{i \varphi}\right)\right| & e^{-\sigma r|\sin \varphi|} d r=\int_{0}^{+\infty}\left|\Xi\left(r e^{i \varphi}\right)\right| d r \leqslant \int_{0}^{+\infty}\left|\int_{0}^{+\infty} f_{1}(i v) \chi\left(i v ; r e^{i \varphi}\right) d v\right| d r \\
& +\int_{0}^{+\infty}\left|\int_{-\infty}^{0} f_{3}(i v) \chi\left(i v ; r e^{i \varphi}\right) d v\right| d r+\int_{0}^{+\infty}\left|\int_{0}^{+\infty} f_{2}(u) \chi\left(u ; r e^{i \varphi}\right) d u\right| d r \\
& \leqslant \int_{0}^{+\infty} \int_{0}^{+\infty}\left|f_{1}(i v) \chi\left(i v ; r e^{i \varphi}\right)\right| d r d v+\int_{-\infty}^{+\infty} \int_{0}^{+\infty}\left|f_{3}(i v) \chi\left(i v ; r e^{i \varphi}\right)\right| d r d v \\
& +\int_{0}^{+\infty} \int_{0}^{+\infty}\left|f_{2}(u) \chi\left(u ; r e^{i \varphi}\right)\right| d r d u .
\end{aligned}
$$


It is clear that

$$
\frac{\pi}{2} \int_{0}^{+\infty}\left|\chi\left(i v ; r e^{i \varphi}\right)\right| d r=\int_{0}^{+\infty} \frac{|v| r \cos \varphi d r}{\left(v^{2}-2 v r \sin \varphi+r^{2}\right) \sqrt{v^{2}+2 v r \sin \varphi+r^{2}}} d r
$$

If $v>0$, then

$$
\int_{0}^{+\infty} \frac{|v| r \cos \varphi d r}{\left(v^{2}-2 v r \sin \varphi+r^{2}\right) \sqrt{v^{2}+2 v r \sin \varphi+r^{2}}}=\int_{0}^{+\infty} \frac{s \cos \varphi d s}{\left(1-2 s \sin \varphi+s^{2}\right) \sqrt{1+2 s \sin \varphi+s^{2}}} .
$$

Since $\varphi \in(0 ; \pi / 2)$, employing inequality $s<\sqrt{s^{2}+1}$, we have

$$
\int_{0}^{+\infty} \frac{s \cos \varphi d s}{\left(1-2 s \sin \varphi+s^{2}\right) \sqrt{1+2 s \sin \varphi+s^{2}}} \leq \int_{0}^{+\infty} \frac{\cos \varphi d s}{1-2 s \sin \varphi+s^{2}}=\frac{\pi}{2}+\arctan \tan \varphi \leq \pi .
$$

If $v<0$, then

$$
\begin{aligned}
& \int_{0}^{+\infty} \frac{|v| r \cos \varphi d r}{\left(v^{2}-2 v r \sin \varphi+r^{2}\right) \sqrt{v^{2}+2 v r \sin \varphi+r^{2}}}=\int_{0}^{+\infty} \frac{s \cos \varphi d s}{\left(1+2 s \sin \varphi+s^{2}\right) \sqrt{1-2 s \sin \varphi+s^{2}}} \\
& \quad \leq \int_{0}^{+\infty} \frac{s \cos \varphi d s}{\left(1+s^{2}\right) \sqrt{2 s(1-\sin \varphi)}}=\int_{0}^{+\infty} \frac{s 2 \sin \left(\frac{\pi}{4}-\frac{\varphi}{2}\right) \cos \left(\frac{\pi}{4}-\frac{\varphi}{2}\right) d s}{\left(1+s^{2}\right) \sqrt{4 s \sin ^{2}\left(\frac{\pi}{4}-\frac{\varphi}{2}\right)}} \\
& \quad \leq \int_{0}^{+\infty} \frac{\sqrt{s} \cos \left(\frac{\pi}{4}-\frac{\varphi}{2}\right) d s}{1+s^{2}} \leq \int_{0}^{+\infty} \frac{\sqrt{s} d s}{1+s^{2}}=\pi \frac{\sqrt{2}}{2} .
\end{aligned}
$$

Thus, by Fubini theorem for $\varphi \in(0 ; \pi / 2)$ we obtain

$$
\int_{0}^{+\infty} d r \int_{0}^{+\infty}\left|f_{1}(i v) \chi\left(i v ; r e^{i \varphi}\right)\right| d v \leq c<+\infty, \quad \int_{0}^{+\infty} d r \int_{0}^{+\infty}\left|f_{3}(i v) \chi\left(i v ; r e^{i \varphi}\right)\right| d v \leq c<+\infty
$$

For $u>0$ we also get

$$
\begin{aligned}
\frac{\pi}{2} \int_{0}^{+\infty}\left|\chi\left(u ; r e^{i \varphi}\right)\right| d r & =\int_{0}^{+\infty} \frac{u x}{|(u+\bar{z})(u-z)(u+z)|} d r \\
& =\int_{0}^{+\infty} \frac{u r \cos \varphi}{\left(u^{2}+2 u r \cos \varphi+r^{2}\right) \sqrt{u^{2}-2 u r \cos \varphi+r^{2}}} d r \\
& =\int_{0}^{+\infty} \frac{s \cos \varphi}{\left(s^{2}+2 s \cos \varphi+1\right) \sqrt{s^{2}-2 s \cos \varphi+1}} d r \\
& =\int_{0}^{2} \frac{2}{\sqrt{s^{2}-2 s \cos \varphi+1}} d r+\int_{2}^{+\infty} \frac{1}{\left(s^{2}+1\right) \sqrt{(s-\cos \varphi)^{2}+\sin ^{2} \varphi}} d r \\
& \leqslant 2 \int_{0}^{2} \frac{1}{\sqrt{(s-\cos \varphi)^{2}+\sin ^{2} \varphi}} d r+\int_{2}^{+\infty} \frac{1}{s \sqrt{2(s-\cos \varphi) \sin \varphi}} d r
\end{aligned}
$$




$$
\leqslant 2 \int_{0}^{2} \frac{1}{|\sin \varphi|} d r+\frac{1}{\sqrt{2 \sin \varphi}} \int_{2}^{+\infty} \frac{1}{(s-\cos \varphi)^{3 / 2}} d r<c_{2} .
$$

Thus, we arrive at

$$
\int_{0}^{+\infty} d r \int_{0}^{+\infty}\left|f_{2}(u) \chi\left(u ; r e^{i \varphi}\right)\right| d u \leq c_{1}<+\infty, \quad \varphi \in(-\pi / 2+\delta ; \pi / 2-\delta) .
$$

Consider case $-\pi / 2<\varphi<0$. We have

$$
\begin{aligned}
& \int_{0}^{+\infty}\left|f\left(r e^{i \varphi}\right)\right| e^{-\sigma r|\sin \varphi|} d r \leqslant \int_{0}^{+\infty}\left|\Xi\left(r e^{i \varphi}\right)\right| d r+\int_{0}^{+\infty}\left|f_{2}\left(r e^{i \varphi}\right)\right| e^{-2 \sigma r|\sin \varphi|} d r \\
& \leqslant \int_{0}^{+\infty}\left|f_{1}(i v)\right| \int_{0}^{+\infty}\left|\chi\left(i v ; r e^{i \varphi}\right)\right| d r d v+\int_{-\infty}^{0}\left|f_{3}(i v)\right| \int_{0}^{+\infty}\left|\chi\left(i v ; r e^{i \varphi}\right)\right| d r d v \\
&+\int_{0}^{+\infty}\left|f_{2}(u)\right| \int_{0}^{+\infty}\left|\chi\left(u ; r e^{i \varphi}\right)\right| d r d u+c .
\end{aligned}
$$

By analogy with the case $0<\varphi<\pi / 2$ one can show that if $v>0$, then

$$
\int_{0}^{+\infty} \frac{|v| r \cos \varphi d r}{\left(v^{2}-2 v r \sin \varphi+r^{2}\right) \sqrt{v^{2}+2 v r \sin \varphi+r^{2}}} \leq \pi \frac{\sqrt{2}}{2}
$$

while if $v>0$, then

$$
\int_{0}^{+\infty} \frac{|v| r \cos \varphi d r}{\left(v^{2}+2 v r \sin \varphi+r^{2}\right) \sqrt{v^{2}-2 v r \sin \varphi+r^{2}}} \leq \pi
$$

Taking into consideration also $(3)$, we prove $(1)$ for $\varphi \in(-\pi / 2 ;-\delta)$.

Proof of Theorem 3 follows from Lemmata 1-3 if one takes into consideration that function $f$ introduced in Lemma 2 is analytic in $\mathbb{C}_{+}$. Indeed, it is obvious that the function

$$
\frac{1}{2 \pi} \int_{0}^{+\infty} \frac{f_{1}(i v)}{i v-z} d v+\frac{1}{2 \pi} \int_{-\infty}^{0} \frac{f_{3}(i v)}{i v-z} d v
$$

is analytic in $\mathbb{C}_{+}$. Function

$$
\frac{1}{2 \pi i} \int_{0}^{+\infty} \frac{f_{2}(u)}{u-z} d u-f_{2}(z)
$$

is analytic in the angles $\mathbb{C}(0 ; \pi / 2)$ and $\mathbb{C}(-\pi / 2 ; 0)$. On the positive real semi-axis the boundary values from the upper and the lower angle coincide by Sochocki theorem. Hence, as in the proof of Lemma 8 in [7], we obtain easily that $f$ is analytic in $\mathbb{C}_{+}$. 


\section{BIBLIOGRAPHY}

1. A.M. Sedleckili. An equivalent definition of $H^{p}$ spaces in the half-plane and some applications // Matem. Sbornik. 1975. V. 96, No. 1. P. 75-82. [Math. USSR Sb. 1975. V. 25, No. 1. P. 69-76.]

2. P. Koosis. Introduction to $H^{p}$ spaces. With an appendix on Wolff's proof of the Corona theorem. Cambridge University Press, Cambridge, 1980.

3. J.B. Garnett. Bounded analytic functions. Academic Press, New York, 1981.

4. B.V. Vinnitskii. On zeros of functions analytic in a half plane and completeness of systems of exponents // Ukrain. Matem. Zhurn. 1994. V. 46, No. 5. P. 484-500. [Ukrainian Math. J. 1994. V. 46, No. 5. P. 514-532.]

5. B.V. Vinnitskii. An extention of Paley-Wiener theorem // Matematychni Studii. 1995. V. 4. P. 37-44. (in Ukrainian.)

6. B. Vinnitskii, V. Dil'nyi A generalization of the Beurling-Lax theorem // Matem. Zametki. 2006. V. 79, No. 3. P. 362-368. [Math. Notes. 2006. V. 79, No. 3-4. P. 335-341.]

7. V. Dilnyi On cyclic functions in weighted hardy spaces // J. Math. Phys. Anal. Geom. 2011. V. 7 , No. 1. P. 19-33.

Bogdan Vasil'evich Vinnitskii,

Drohobych state pedagogical university named after Ivan Franko,

Stryiskaya str., 3

82100, Drohobych, Ukraine

Vladimir Nikolaevich Dilnyi,

Ivan Franko National University of Lviv,

Universytetska str., 1 ,

82000, Lviv, Ukraine

E-mail: dilnyi@mail.ru 